\title{
Generalized Tesler matrices, virtual Hilbert series, and Macdonald polynomial operators
}

\author{
Andrew Timothy Wilson $\|^{\dagger}$
}

UC San Diego, La Jolla, CA, USA

\begin{abstract}
We generalize previous definitions of Tesler matrices to allow negative matrix entries and non-positive hook sums. Our main result is an algebraic interpretation of a certain weighted sum over these matrices. Our interpretation uses virtual Hilbert series, a new class of symmetric function specializations which are defined by their values on (modified) Macdonald polynomials. As a result of this interpretation, we obtain a Tesler matrix expression for the Hall inner product $\left\langle\Delta_{f} e_{n}, p_{1 n}\right\rangle$, where $\Delta_{f}$ is a symmetric function operator from the theory of diagonal harmonics. We use our Tesler matrix expression, along with various facts about Tesler matrices, to provide simple formulas for $\left\langle\Delta_{e_{1}} e_{n}, p_{1^{n}}\right\rangle$ and $\left.\left\langle\Delta_{e_{k}} e_{n}, p_{1^{n}}\right\rangle\right|_{t=0}$ involving $q, t$-binomial coefficients and ordered set partitions, respectively.

Résumé. Nous généralisons les définitions précédentes de matrices Tesler pour permettre les entrées de la matrice négatives et des montants crochet non-positifs. Notre principal résultat est une interprétation algébrique d'une certaine somme pondérée sur ces matrices. Notre interprétation utilise série de Hilbert virtuel, une nouvelle classe de spécialisations fonctionnelles symétriques qui sont définies par leurs valeurs sur les polynômes de Macdonald (modifiées). À la suite de cette interprétation, on obtient une expression de la matrice Tesler pour la salle intérieure produit $\left\langle\Delta_{f} e_{n}, p_{1^{n}}\right\rangle$, où $\Delta_{f}$ est un opérateur de fonction symétrique de la théorie harmoniques de diagonale. Nous utilisons notre expression de la matrice Tesler, ainsi que divers faits sur des matrices Tesler, de fournir des formules simples pour $\left\langle\Delta_{e_{1}} e_{n}, p_{1^{n}}\right\rangle$ et $\left.\left\langle\Delta_{e_{k}} e_{n}, p_{1^{n}}\right\rangle\right|_{t=0}$ impliquant $q, t$-coefficients binomial et ensemble ordonné partitions, respectivement.
\end{abstract}

Keywords: Tesler matrices, Macdonald polynomials, Shuffle Conjecture, ordered set partitions

\section{Introduction}

Given a vector $\alpha \in \mathbb{Z}^{n}$, we define the Tesler matrices with hook sums $\alpha$ to be the set of all $n$ by $n$ matrices $A$ with entries in $\mathbb{Z}$ such that

1. $A$ is upper triangular,

2. A has no zero rows,

3. each row of $A$ is either entirely non-negative or entirely non-positive, and

\footnotetext{
${ }^{\dagger}$ Email: atwilsoneucsd. edu. Partially supported by an NDSEG Fellowship. 
4. the kth hook sum of $A$, defined by

$$
\left(a_{k, k}+a_{k, k+1}+\ldots a_{k, n}\right)-\left(a_{k, 1}+a_{k, 2}+\ldots a_{k, k-1}\right)
$$

equals $\alpha_{k}$ for every $1 \leq k \leq n$.

We will sometimes refer to a matrix that satisfies condition 2 as essential and a matrix that satisfies condition 3 as signed. Since previous work on Tesler matrices primarily addresses matrices with positive hook sums, and conditions 2 and 3 are trivial in that setting, our definition generalizes previous definitions of Tesler matrices. We denote the set of Tesler matrices with hook sums $\alpha$ by $\mathcal{T}(\alpha)$.

The cases $\alpha=(1,1, \ldots, 1)$ and $\alpha=(1, m, \ldots, m)$ for any positive integer $m$ are studied in [Hag11], where they are used to give an expression for the Hilbert series of the (generalized) module of diagonal harmonics. More values of $\alpha$ have appeared in the study of Hall-Littlewood polynomials $\mathrm{AGR}^{+} 12$, Macdonald polynomial operators [GHX14], and flow polytopes [MMR14]. It would be particularly interesting to see which methods from [MMR14] can be extended to our (essential, signed) Tesler matrices.

We set the weight of an $n$ by $n$ Tesler matrix $A$ to be

$$
\operatorname{wt}(A)=(-1)^{\text {entries }^{+}(A)-\operatorname{rows}^{+}(A)} M^{\operatorname{nonzero}(A)-n} \prod_{a_{i, j} \neq 0}\left[a_{i, j}\right]_{q, t}
$$

where $M=(1-q)(1-t)$, entries ${ }^{+}(A)$ is the number of positive entries in $A$, rows ${ }^{+}(A)$ is the number of rows of $A$ whose nonzero entries are all positive, nonzero $(A)$ is the number of nonzero entries of $A$, and $[k]_{q, t}=\frac{q^{k}-t^{k}}{q-t}$, the usual $q, t$-analogue of an integer $k$. Since $A$ is essential, the exponent of $M$ is nonnegative and $\operatorname{wt}(A) \in \mathbb{Z}[q, t, 1 / q, 1 / t]$. When $A$ has no negative entries, this weight function is equal to the weight function defined in [Hag11]. It is also worth noticing that the weight of a Tesler matrix is independent of $\alpha$. We define the Tesler polynomial with hook sums $\alpha$ to be

$$
\operatorname{Tes}(\alpha ; q, t)=\sum_{A \in \mathcal{T}(\alpha)} \operatorname{wt}(A)
$$

In [Hag11], Haglund showed that $\operatorname{Tes}\left(1^{n} ; q, t\right)$ is equal to the Hilbert series of the module of diagonal harmonics, which can also be written in terms of Macdonald polynomial operators as $\left\langle\nabla e_{n}, p_{1^{n}}\right\rangle$ or $\left\langle\Delta_{e_{n}} e_{n}, p_{1^{n}}\right\rangle$. [GHX14] contains an algebraic interpretation for $\operatorname{Tes}(\alpha ; q, t)$ for any $\alpha$ with positive integer entries. We summarize these results, along with the necessary notation, in Section 2

In Section 3 , we develop an algebraic interpretation for $\operatorname{Tes}(\alpha ; q, t)$ for any $\alpha \in \mathbb{Z}^{n}$ in terms of new symmetric function specializations which we call virtual Hilbert series. Our interpretation is equivalent to the interpretation in [GHX14] for positive hook sums; in this sense, the Tesler matrix definition we have used here is the natural extension of previous definitions. These specializations generalize the map that sends a symmetric function $f$ that is homogeneous of degree $n$ to its inner product with $p_{1^{n}}$. In the case that $f$ is the Frobenius image of an $\mathfrak{S}_{n}$-module, this inner product extracts the module's Hilbert series. With this in mind, for any symmetric function $f$ that is homogenous of degree $n$, we will often use the notation

$$
\text { Hilb } f=\left\langle f, p_{1^{n}}\right\rangle \text {. }
$$




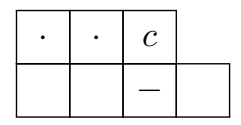

Fig. 1: This is the Young diagram (in French notation) of the partition $(4,3)$. The cell $c$ has $a^{\prime}(c)=2$ (represented by dots) and $l^{\prime}(c)=1$ (represented by dashes).

In Section 4, we show that certain sums of virtual Hilbert series appear in the study of diagonal harmonics, especially in connection with the Macdonald polynomial operators $\Delta_{f}$ and modified versions of these operators, which we denote $\Delta_{f}^{\prime}$. We use the algebraic interpretation of Tesler polynomials from Section 3 to produce a number of new results about these operators.

Specifically, in Subsection 4.1 we obtain a Tesler polynomial expression for Hilb $\Delta_{f}^{\prime} e_{n}$ for any symmetric Laurent polynomial $f$. This expression leads directly to a similar expression for Hilb $\Delta_{f} e_{n}$. If $f$ has coefficients in $\mathbb{Z}[q, t]$, then our formula is clearly in $\mathbb{Z}[q, t, 1 / q, 1 / t]$. Moreover, if $f$ is a symmetric function, then our formula is in $\mathbb{Z}[q, t]$. These results provide direct formulas for the Hilbert series case of Theorem 1.3 in [BGHT99].

Subsection 4.2 contains a formula for Hilb $\Delta_{e_{1}} e_{n}$ that directly implies Hilb $\Delta_{e_{1}} e_{n} \in \mathbb{N}[q, t]$, which is not otherwise obvious as there is currently no module associated with $\Delta_{e_{1}} e_{n}$.

In Subsection 4.3 we show how Hilb $\Delta_{e_{k}}^{\prime} e_{n}$ at $t=0$ is related to statistics on ordered set partitions, generalizing [Lev12]. This result completes the proof of a special case of a generalization of the Shuffle Conjecture due to Haglund [Hag14].

\section{Background}

First, we fix some notation. We use $\Lambda$ to denote the algebra of symmetric functions over the base field $\mathbb{Q}(q, t)$. If we wish to refer to the elements of $\Lambda$ which are homogeneous of degree $n$, we will write $\Lambda^{n}$. Similarly, we will use $\underline{\Lambda}$ and $\underline{\Lambda}^{n}$ to refer to the algebra of symmetric Laurent polynomials. Occasionally, we will use $\mathbb{Z}[q, t]$ as a subscript to refer to the subalgebra of symmetric functions or symmetric Laurent polynomials consisting of the functions with coefficients in $\mathbb{Z}[q, t]$.

There are several important classical bases for the space of symmetric functions (viewed as a vector space): the monomial symmetric functions $\left\{m_{\lambda}\right\}$, the elementary symmetric functions $\left\{e_{\lambda}\right\}$, the homogeneous symmetric functions $\left\{h_{\lambda}\right\}$, the power symmetric functions $\left\{p_{\lambda}\right\}$, and the Schur functions $\left\{s_{\lambda}\right\}$. The (modified) Macdonald polynomials $\left\{\tilde{H}_{\lambda}\right\}$ are also a basis for this space, and generalize many of the important properties of the classical bases. We refer the reader to [Sta99, Mac95] for more material on symmetric functions and Macdonald polynomials. The only basis we will use for the symmetric Laurent polynomials is the monomial basis $\left\{\underline{m}_{\rho}\right\}$, defined as the sum of all monomials whose exponents, when arranged in weakly decreasing order, equal the finite, weakly decreasing vector of nonzero integers $\rho$. We will refer to a finite vector of weakly decreasing nonzero integers as a Laurent partition.

When studying Macdonald polynomials, it is quite useful to have the following notation. Given a partition $\mu \vdash n$ and a cell $c$ in the Young diagram of $\mu$ (drawn in French notation) we set $a^{\prime}(c)$ and $l^{\prime}(c)$ to be the number of cells in $\mu$ that are strictly to the left and strictly below $c$ in $\mu$, respectively. In Figure 1 we compute these values for a particular example. 
We set

$$
T_{\mu}=\prod_{c \in \mu} q^{a^{\prime}(c)} t^{l^{\prime}(c)} \quad B_{\mu}=\sum_{c \in \mu} q^{a^{\prime}(c)} t^{l^{\prime}(c)}
$$

We will use this notation to define a number of operators on $\Lambda^{n}$. Each of the operators is defined by its action on the Macdonald polynomial basis. First, we set

$$
\nabla \tilde{H}_{\mu}=T_{\mu} \tilde{H}_{\mu} .
$$

The $\nabla$ operator has become quite famous due to its connection with the module of diagonal harmonics. Specifically, in [Hai02] Haiman proved that the Frobenius image of the character of the module of diagonal harmonics of order $n$ is equal to $\nabla e_{n}$. For more on this module, see [Hag08]. The main result in [Hag11] is that

$$
\operatorname{Hilb} \nabla e_{n}=\operatorname{Tes}\left(1^{n} ; q, t\right) .
$$

Given any symmetric Laurent polynomial $f$, we define two more operators on $\Lambda^{n}$ by

$$
\Delta_{f} \tilde{H}_{\mu}=f\left[B_{\mu}\right] \tilde{H}_{\mu} \quad \Delta_{f}^{\prime} \tilde{H}_{\mu}=f\left[B_{\mu}-1\right] \tilde{H}_{\mu} .
$$

Here, we have used the notation that, for a symmetric Laurent polynomial $f$ and a sum $S=s_{1}+\ldots+s_{k}$ of monic monomials, $f[S]$ is equal to the specialization of $f$ at $x_{1}=s_{1}, \ldots, x_{k}=s_{k}$, where the remaining variables are set equal to zero. Note that both operators are linear in their subscripts. The operator $\Delta_{f}$, at least in the case where $f$ is a symmetric function, appears often in the study of diagonal harmonics [Hag08]. Furthermore, it is clear that $\Delta_{e_{n}}=\nabla$ when applied to $\Lambda^{n}$, so $\nabla e_{n}=\Delta_{e_{n}} e_{n}$. Although the operator $\Delta_{f}^{\prime}$ is not as common, it can be connected to $\Delta_{f}$ via the identity

$$
\Delta_{\underline{m}_{\rho}}=\Delta_{\underline{m}_{\rho}}^{\prime}+\sum_{\nu \in \widehat{\rho}} \Delta_{\underline{m}_{\nu}}^{\prime}
$$

where $\widehat{\rho}$ is defined to be the set of all Laurent partitions which can be obtained by removing one part from $\rho$. In particular,

$$
\Delta_{e_{k}}=\Delta_{e_{k}}^{\prime}+\Delta_{e_{k-1}}^{\prime}
$$

which, combined with the fact that $\Delta_{f}^{\prime} g=0$ if the degree of $f$ is greater than or equal to the degree of $g$, implies $\nabla e_{n}=\Delta_{e_{n-1}}^{\prime} e_{n}$. We allow for $f$ to be a symmetric Laurent polynomial because it provides a way to obtain negative powers of $\nabla$ in terms of our operators via the identity

$$
\nabla^{-1}=\Delta_{\underline{m}_{(-1)^{n}}}
$$

on $\Lambda^{n}$.

We will also make use of the skew Pieri coefficients of $\tilde{H}_{\mu}$. We define the skewing operator on $\Lambda$ by insisting that

$$
\left\langle f^{\perp} g, h\right\rangle=\langle g, f h\rangle
$$


for any symmetric functions $f, g$, and $h$. Here and in the sequel, we use the Hall inner product on symmetric functions. Then the skew Pieri coefficients $c_{\mu, \nu}$ are defined by

$$
e_{1}^{\perp} \tilde{H}_{\mu}=\sum_{\nu \rightarrow \mu} c_{\mu, \nu} \tilde{H}_{\nu}
$$

where the sum is over all partitions $\nu$ that can be obtained by removing a single cell from $\mu$. In [GHX14], the authors use a constant term algorithm to provide a formula for $\operatorname{Tes}(\alpha ; q, t)$ for any vector $\alpha$ of positive integers in terms of the skewing operator.

Finally, we will employ the following standard notation for $q, t$ - and $q$-analogues of integers:

$$
\begin{aligned}
{[n]_{q, t} } & =\frac{q^{n}-t^{n}}{q-t} \\
{[n]_{q} } & =[n]_{q, 1}=\frac{q^{n}-1}{q-1} .
\end{aligned}
$$

Note that $[n]_{q, t} \in \mathbb{N}[q, t]$ if $n \geq 0$ and $[n]_{q, t} \in \mathbb{Z}[1 / q, 1 / t]$ if $n \leq 0$. This implies Tes $(\alpha ; q, t) \in$ $\mathbb{Z}[q, t, 1 / q, 1 / t]$ for any $\alpha \in \mathbb{Z}^{n}$.

\section{Virtual Hilbert Series}

In this section, we use new symmetric function specializations to derive an algebraic interpretation for $\operatorname{Tes}(\alpha ; q, t)$ for any vector of integers $\alpha$. Our interpretation generalizes the formulas in [Hag11, GHX14]. Given any $\alpha \in \mathbb{Z}^{n-1}$ and $\mu \vdash n$, we make the following recursive definition.

$$
\begin{aligned}
F_{\mu}^{\alpha} & =\sum_{\nu \rightarrow \mu} c_{\mu, \nu}\left(T_{\mu} / T_{\nu}\right)^{\alpha_{1}} F_{\nu}^{\left(\alpha_{2}, \ldots, \alpha_{n-1}\right)} \\
F_{(1)}^{()} & =1
\end{aligned}
$$

It is worth noting that $F_{\mu}^{0^{n-1}}=$ Hilb $\tilde{H}_{\mu}$, the Hilbert series of the Garsia-Haiman module associated with $\tilde{H}_{\mu}$, which is sometimes denoted $F_{\mu}$. As a result, $F_{\mu}^{\alpha}$ can be thought of as a generalization of this Hilbert series. The famous $n$ ! conjecture of Garsia and Haiman, proved in [Hai01], is simply the statement that setting $q=t=1$ in $F_{\mu}$ yields $n$ !. We list some open questions about the $F_{\mu}^{\alpha}$ below.

- Computations in Sage suggest that $F_{\mu}^{\alpha}$ is always a polynomial. Is this true?

- For which $\alpha, \mu$ is $F_{\mu}^{\alpha} \in \mathbb{N}[q, t]$ ?

- HHL05a gives a combinatorial formula for $F_{\mu}$. Is there a similar combinatorial formula for $F_{\mu}^{\alpha}$ ?

Now we define a map

$$
\begin{aligned}
& \operatorname{Hilb}_{\alpha}: \Lambda^{n} \rightarrow \mathbb{Q}(q, t) \\
& \tilde{H}_{\mu} \mapsto F_{\mu}^{\alpha}
\end{aligned}
$$


We will sometimes refer to $\operatorname{Hilb}_{\alpha} f$ as the virtual Hilbert series of $f$ with respect to $\alpha$. We can justify this terminology by noting that $F_{\mu}^{0^{n-1}}=F_{\mu}$ implies

$$
\text { Hilb }_{0^{n-1}}=\text { Hilb } .
$$

Furthermore, we have

$$
\operatorname{Hilb}_{m^{n-1}}=\operatorname{Hilb} \nabla^{m} \text {. }
$$

for any integer $m$. The following result gives an algebraic interpretation for $\operatorname{Tes}(\alpha ; q, t)$ for any $\alpha \in \mathbb{Z}^{n-1}$. We note that the right-hand side is equivalent to the right-hand side of I.9 in [GHX14].

Theorem 3.1. For any $\alpha \in \mathbb{Z}^{n-1}$, we have

$$
\operatorname{Tes}(\alpha ; q, t)=\frac{(-1)^{n-1}}{[n]_{q}[n]_{t}} \operatorname{Hilb}_{\alpha} p_{n}
$$

If we are willing to restrict our attention to vectors that begin with a 1, we can simplify the right-hand side of Theorem 3.1] slightly. We also obtain a direct generalization of the results in [Hag11].

Corollary 3.1. For any $\alpha \in \mathbb{Z}^{n-1}$, we have

$$
\operatorname{Tes}((1, \alpha) ; q, t)=\operatorname{Hilb}_{\alpha} e_{n} .
$$

\section{Applications to Delta Operators}

The right-hand sides of Theorem 3.1 and Corollary 3.1 bear some similarity to symmetric function expressions popular in the study of diagonal harmonics. In this section, we explore these connections and use the connections to prove new results about the Macdonald polynomial operators $\Delta_{f}$ and $\Delta_{f}^{\prime}$.

\subsection{Polynomial Expressions}

Recall that we have defined an operator $\Delta_{f}^{\prime}$ on $\Lambda^{n}$ by stating that it acts on the Macdonald polynomials by

$$
\Delta_{f}^{\prime} \tilde{H}_{\mu}=f\left[B_{\mu}-1\right] \tilde{H}_{\mu}
$$

Although we will not be able to describe every virtual Hilbert series in terms of this operator, we do have the following result involving certain symmetric sums of virtual Hilbert series. We let sort be the map that removes the zeros from $\alpha$ and then sorts the remaining entries in weakly decreasing order.

Theorem 4.1. For any Laurent partition $\rho$,

$$
\operatorname{Hilb} \Delta_{\underline{m}_{\rho}}^{\prime}=\sum_{\alpha: \operatorname{sort}(\alpha)=\rho} \operatorname{Hilb}_{\alpha}
$$

Using the Tesler polynomial expressions obtained in Section 3 , we obtain the following identities. 


\section{Corollary 4.1.}

$$
\begin{aligned}
\frac{(-1)^{n-1}}{[n]_{q}[n]_{t}} \text { Hilb } \Delta_{\underline{m}_{\rho}}^{\prime} p_{n} & =\sum_{\alpha: \operatorname{sort}(\alpha)=\rho} \operatorname{Tes}(\alpha ; q, t) \\
\operatorname{Hilb} \Delta_{\underline{m}_{\rho}}^{\prime} e_{n} & =\sum_{\alpha: \operatorname{sort}(\alpha)=\rho} \operatorname{Tes}((1, \alpha) ; q, t)
\end{aligned}
$$

As a result, both left-hand sides are in $\mathbb{Z}[q, t, 1 / q, 1 / t]$. Furthermore, by the linearity in the subscript of $\Delta_{f}^{\prime}$ we have

$$
\begin{array}{r}
\frac{(-1)^{n-1}}{[n]_{q}[n]_{t}} \text { Hilb } \Delta_{f}^{\prime} p_{n}, \text { Hilb } \Delta_{f}^{\prime} e_{n} \in \mathbb{Z}[q, t, 1 / q, 1 / t] \\
\frac{(-1)^{n-1}}{[n]_{q}[n]_{t}} \text { Hilb } \Delta_{g}^{\prime} p_{n}, \text { Hilb } \Delta_{g}^{\prime} e_{n} \in \mathbb{Z}[q, t]
\end{array}
$$

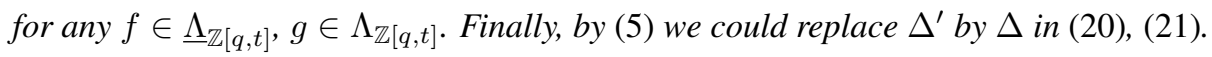

Corollary 4.1 can be thought of as a more concrete version of a special case of Theorem 1.3 in [BGHT99], in which the authors showed that $\Delta_{f} \Lambda_{\mathbb{Z}[q, t]} \subseteq \Lambda_{\mathbb{Z}[q, t]}$ for any $f \in \Lambda_{\mathbb{Z}[q, t]}$. Corollary 4.1 provides the first direct formulas for Hilbert series of expressions of this type.

It may be of interest to the reader to use Corollary 4.1 in order to explicitly compute some Hilb $\Delta_{f} e_{n}$. Rather than state the exact analogue of 19 ) for this case, we mention that the following process accomplishes this task.

1. Expand $f$ into variables $x_{1}, x_{2}, \ldots, x_{n-1}, 1$.

2. Replace each monomial $x^{\alpha}$ in this expansion with $\operatorname{Tes}((1, \alpha) ; q, t)$.

As an example, we compute Hilb $\Delta_{s_{3,2,1}} e_{3}$.

$$
s_{3,2,1}\left(x_{1}, x_{2}, 1\right)=x_{1}^{3} x_{2}^{2}+x_{1}^{2} x_{2}^{3}+x_{1}^{3} x_{2}+2 x_{1}^{2} x_{2}^{2}+x_{1} x_{2}^{3}+x_{1}^{2} x_{2}+x_{1} x_{2}^{2} .
$$

Replacing each monomial with its associated Tesler polynomial, we get

$$
\begin{aligned}
\text { Hilb } \Delta_{s_{3,2,1}} e_{3}= & \operatorname{Tes}((1,3,2) ; q, t)+\operatorname{Tes}((1,2,3) ; q, t)+\operatorname{Tes}((1,3,1) ; q, t) \\
& +2 \operatorname{Tes}((1,2,2) ; q, t)+\operatorname{Tes}((1,1,3) ; q, t) \\
& +\operatorname{Tes}((1,2,1) ; q, t)+\operatorname{Tes}((1,1,2) ; q, t) .
\end{aligned}
$$

We have not explored how this method compares to current methods for computing Hilb $\Delta_{f} e_{n}$ from a computational perspective.

\subsection{Positive Formulas}

In this subsection, we use Corollary 4.1 to obtain formulas for Hilb $\Delta_{e_{1}} e_{n}$, Hilb $\frac{(-1)^{n-1}}{[n]_{q}[n]_{t}} \operatorname{Hilb} \Delta_{e_{2}} p_{n}$, and Hilb $\Delta_{m_{-1}} e_{n}$. Each formula shows that the Hilbert series of the given symmetric function is positive with respect to some set of variables. The operator $\Delta_{e_{1}}$ can be thought of as a translation of Macdonald's original $E$ operator in [Mac95] to the context of the modified Macdonald polynomials $\tilde{H}_{\mu}$. 


\section{Corollary 4.2.}

$$
\begin{aligned}
\text { Hilb } \Delta_{e_{1}} e_{n} & =\sum_{k=1}^{n}\left(\begin{array}{l}
n \\
k
\end{array}\right)[k]_{q, t} \\
\frac{(-1)^{n-1}}{[n]_{q}[n]_{t}} \text { Hilb } \Delta_{e_{2}} p_{n} & =\sum_{k=1}^{n-1}\left(\begin{array}{c}
n-1 \\
k
\end{array}\right)[k]_{q, t} \\
\text { Hilb } \Delta_{m_{-1}} e_{n} & =\left(1-\frac{1}{q t}\right)^{n-1} .
\end{aligned}
$$

As a result, the left-hand sides of (26) and [27] are in $\mathbb{N}[q, t]$ and the left-hand side of [28] is in $\mathbb{N}\left[(q t)^{-1}\right]$.

In forthcoming work of the author with Haglund and Remmel, we use a reciprocity identity to give the full Schur expansion of $\Delta_{e_{1}} e_{n}$, which implies (26). The advantage of our approach here is that it is somewhat more combinatorial.

We sketch the proof of (26), since the proofs of $(27)$ and $(28)$ are quite similar. By $(6)$, the left-hand side of 26 is equal to

$$
\operatorname{Tes}\left(\left(1,0^{n-1}\right) ; q, t\right)+\sum_{i=0}^{n-2} \operatorname{Tes}\left(\left(1,0^{i}, 1,0^{n-i-2}\right) ; q, t\right) .
$$

In order to simplify the right-hand side, we make the following observation about Tesler polynomials.

Lemma 4.1. For any $\alpha \in \mathbb{Z}^{n}$,

$$
\operatorname{Tes}((1, \alpha) ; q, t)=\operatorname{Tes}(\alpha ; q, t)+\sum_{i=1}^{n} \operatorname{Tes}\left(\left(\alpha_{1}, \ldots, \alpha_{i-1}, \alpha_{i}+1, \alpha_{i+1}, \ldots, \alpha_{n}\right) ; q, t\right) .
$$

Proof. Consider a Tesler matrix $A$ with hook sums $(1, \alpha)$. Its first row must consist of a single nonzero entry, which must be equal to 1 . Say this entry occurs in column $j$, i.e. $a_{1, j}=1$. If $j=1$, removing the first row of $j$ produces a Tesler matrix with hook sums $\alpha$, and this process is a weight-preserving bijection. If $j>1$, the process is still a weight-preserving bijection, but now we have produced a Tesler matrix with hook sums $\left(\alpha_{1}, \ldots, \alpha_{j-2}, \alpha_{j-1}+1, \alpha_{j}, \ldots, \alpha_{n}\right)$.

Since $\operatorname{Tes}(\alpha ; q, t)=0$ if $\alpha_{1}=0$, Lemma 4.1 implies 29$]$ is

$$
\begin{aligned}
& =\operatorname{Tes}((1) ; q, t)+\sum_{i=0}^{n-2} \operatorname{Tes}\left(\left(1,1,0^{i}\right) ; q, t\right) \\
& =\operatorname{Hilb} \Delta_{e_{1}} e_{n-1}+\operatorname{Tes}\left(\left(1,1,0^{n-2}\right) ; q, t\right) .
\end{aligned}
$$

Furthermore, applying Lemma 4.1 again, we get

$$
\begin{aligned}
\operatorname{Tes}\left(\left(1,1,0^{n-2}\right) ; q, t\right) & =\operatorname{Tes}\left(\left(2,0^{n-2}\right) ; q, t\right)+\operatorname{Tes}\left(\left(1,1,0^{n-3}\right) ; q, t\right)+\ldots+\operatorname{Tes}\left(\left(1,0^{n-3}, 1\right) ; q, t\right) \\
& =\operatorname{Tes}\left(\left(2,0^{n-2}\right) ; q, t\right)+\operatorname{Hilb} \Delta_{e_{1}} e_{n-1}-1
\end{aligned}
$$


Therefore

$$
\text { Hilb } \Delta_{e_{1}} e_{n}=2 \operatorname{Hilb} \Delta_{e_{1}} e_{n-1}-1+\operatorname{Tes}\left(\left(2,0^{n-2}\right) ; q, t\right)
$$

Finally, we consider what happens when we remove the first row of a Tesler matrix with hook sums $\left(2,0^{n-2}\right)$. We claim that a detailed analysis of this situation implies

$$
\operatorname{Tes}\left(\left(2,0^{k}\right) ; q, t\right)=[2]_{q} \operatorname{Tes}\left(\left(2,0^{k-1}\right) ; q, t\right)-M \text { Hilb } \Delta_{e_{1}} e_{k}
$$

Iterating this recursion, we obtain

$$
\operatorname{Hilb} \Delta_{e_{1}} e_{n}=2 \operatorname{Hilb} \Delta_{e_{1}} e_{n-1}-2+[2]_{q}^{n-1}-M \sum_{k=1}^{n-2}[2]_{q}^{n-k-2} \operatorname{Hilb} \Delta_{e_{1}} e_{k}
$$

which we can prove satisfies 26 by induction.

\subsection{The $t=0$ case}

In this subsection, we show how to relate Hilb $\left.\Delta_{e_{k}}^{\prime} e_{n}\right|_{t=0}$ to the distribution of an inversion statistic on ordered set partitions studied in [RW13]. Together with [ [RW13], this completes the proof of a new special case of a conjectural combinatorial interpretation for $\Delta_{e_{k}}^{\prime} e_{n}$ made recently by Haglund [Hag14].

First, we set $\mathcal{O P} \mathcal{P}_{n, k}$ to be the ordered partitions of the set $\{1,2, \ldots, n\}$ into exactly $k$ blocks. Given a subset $S$ of $\{1,2, \ldots, n\}$, we set $\mathcal{O} \mathcal{P}_{n, S}$ to be the ordered set partitions in which the minimal elements of the blocks are exactly the elements of $S$. For example, $7|236| 45 \mid 1$ is an element of $\mathcal{O P}_{7,\{1,2,4,7\}}$ and $\mathcal{O P} \mathcal{P}_{7,4}$

The inversion number (inv) of an ordered set partition $\pi$ is defined to be the number of pairs $(i, j)$ with $i>j$ such that

- $i$ 's block is strictly to the left of $j$ 's block in $\pi$, and

- $j$ is the smallest element in its block in $\pi$.

For example, $\operatorname{inv}(7|236| 45 \mid 1)=9$.

Investigating the connection between virtual Hilbert series and Tesler polynomials at $t=0$, we obtain the following corollary.

Corollary 4.3. For any $\beta \in\{0,1\}^{n-1}$, set

$$
S(\beta)=\{1\} \cup\left\{i+1: \beta_{i}=1\right\} .
$$

Then we have

$$
\begin{aligned}
\left.\operatorname{Hilb}_{\beta} e_{n}\right|_{t=0} & =\sum_{\pi \in \mathcal{O} \mathcal{P}_{n, S(\beta)}} q^{\operatorname{inv}(\pi)} \\
\left.\operatorname{Hilb} \Delta_{e_{k}}^{\prime} e_{n}\right|_{t=0} & =\sum_{\pi \in \mathcal{O} \mathcal{P}_{n, k+1}} q^{\operatorname{inv}(\pi)} .
\end{aligned}
$$


In order to sketch the proof of Corollary 4.3. we first note that, in [Lev12], Levande defined a map from Tesler matrices with hook sums $1^{n}$ to $\mathfrak{S}_{n}$. We will denote this map by $L_{n}$. Furthermore, Levande used a weight-preserving, sign-reversing involution to prove that

$$
\left.\sum_{A: L_{n}(A)=\sigma} \operatorname{wt}(A)\right|_{t=0}=q^{\operatorname{inv}(\sigma)} .
$$

for any $\sigma \in \mathfrak{S}_{n}$. Here inv is the usual inversion number (or Coxeter length) of a permutation, which can be defined as

$$
\operatorname{inv}(\sigma)=\left|\left\{(i, j): 1 \leq i<j \leq n: \sigma_{i}>\sigma_{j}\right\}\right| .
$$

Summing (41) over all permutations $\sigma \in \mathfrak{S}_{n}$ yields

$$
\operatorname{Tes}\left(1^{n} ; q, 0\right)=[n]_{q} !
$$

We extend Levande's results to our setting as follows. For any $\beta \in\{0,1\}^{n-1}$, we define a map $L_{\beta}$ from Tesler matrices with hook sums $(1, \beta)$ onto $\mathcal{O P}{ }_{n, S(\beta)}$. To define $L_{\beta}$, we first map a Tesler matrix $A$ with hook sums $(1, \beta)$ to an intermediary array. This array is created as follows:

1. First, read the entries of the diagonal $a_{j, j}$ for $j=n$ to 1 . If $a_{j, j}>0$, write a $j$ in the rightmost column of the array $a_{j, j}$ times. After this step, the array will have a single column of length $k$ which weakly decreases from top to bottom.

2. For every $j=n$ to 1 , read up the $j$ th column from $a_{j-1, j}$ to $a_{1, j}$. For every $a_{i, j}>0$, find the highest $j$ in the array that currently has no entries to its left. Place an $i$ to its left. Place an $i$ in this manner $a_{i, j}$ times.

For example, we send

$$
\left[\begin{array}{llll}
0 & 0 & 0 & 1 \\
0 & 0 & 1 & 0 \\
0 & 0 & 1 & 0 \\
0 & 0 & 0 & 2
\end{array}\right] \mapsto\left(\begin{array}{cc}
1 & 4 \\
& 4 \\
2 & 3
\end{array}\right) .
$$

Given such an array, we produce an ordered set partition by the following process.

1. Read the leftmost entries in each row of the array from bottom to top. Make these the minimal elements in $k$ different blocks, from left to right.

2. For each $i=1$ to $n$ which is not yet placed into the ordered set partition, find the lowest row in the array in which it appears. Place it in the block which contains the leftmost entry of that row.

Continuing our example, we obtain the ordered set partition $23|4| 1$. We claim that a weight-preserving sign-reversing involution very similar to Levande's proves that

$$
\left.\sum_{A: L_{\beta}(A)=\pi} \operatorname{wt}(A)\right|_{t=0}=q^{\operatorname{inv}(\pi)} .
$$


In forthcoming work of the author with Haglund and Remmel, we combinatorially define a symmetric function ${ }^{(i)} \operatorname{Val}_{n, k}(x ; q, t)$ with coefficients in $\mathbb{N}[q, t]$ and conjecture that

$$
\operatorname{Val}_{n, k}(x ; q, t)=\Delta_{e_{k}}^{\prime} e_{n}
$$

When $k=n-1$ this conjecture reduces to the Shuffle Conjecture [ $\left.\mathrm{HHL}^{+} 05 \mathrm{~b}\right]$. It is evident from the definition of this polynomial that

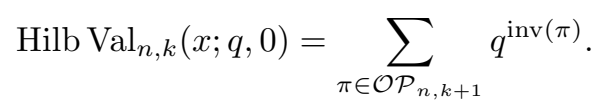

Hence, Corollary 4.3 can be seen as proving the $t=0$ case of the generalization of the Shuffle Conjecture in (45).

\section{Future Work}

In our results so far, we have relied heavily on the fact that we are taking Hilbert series of the various symmetric functions at hand. It is reasonable to ask how Tesler matrices can be used to give formulas for the symmetric functions themselves. For example, in [GH14], Garsia and Haglund use Tesler matrices to give a formula for the symmetric function $\nabla e_{n}$. A similar (but not equivalent) formula for (rational extensions of) $\nabla e_{n}$ is given in [GN13].

In a similar vein, it would be interesting to obtain symmetric functions whose Hilbert series are equal to $F_{\mu}^{\alpha}$. Such a result would allow us to replace virtual Hilbert series with the actual Hilbert series of these symmetric functions.

Finally, it seems possible that the methods used in Subsection 4.2 and 4.3 could be applied when $e_{1}$ is replaced by a slightly more complicated function ( $e_{2}$ or $m_{2}$, for example). Similarly, we may be able to extend the results in Subsection 4.3 to $\beta$ with entries not equal to 0 or 1 . The computations will be more difficult in these cases, but they may still be tractable.

\section{References}

[AGR $\left.{ }^{+} 12\right]$ D. Armstrong, A. Garsia, B. Rhoades, J. Haglund, and B. Sagan. Combinatorics of Tesler matrices in the theory of parking functions and diagonal harmonics. J. Combin., 3:451-494, 2012.

[BGHT99] F. Bergeron, A. M. Garsia, M. Haiman, and G. Tesler. Identities and positivity conjectures for some remarkable operators in the theory of symmetric functions. Meths. and Appls. of Analysis, 6(3):363-420, 1999.

[GH14] A. Garsia and J. Haglund. A polynomial expression for the character of diagonal harmonics. To appear in Ann. of Combin., 2014.

[GHX14] A. Garsia, J. Haglund, and G. Xin. Constant term methods in the theory of Tesler matrices and Macdonald polynomial operators. Ann. Combin., 38:83-109, 2014.

(i) Actually, by definition the function is only quasisymmetric; it is conjecturally a symmetric function. 
[GN13] E. Gorsky and A. Negut. Refined knot invariants and Hilbert schemes. arXiv:1304.3328, April 2013.

[Hag08] J. Haglund. The q, t-Catalan Numbers and the Space of Diagonal Harmonics. Amer. Math. Soc., 2008. Vol. 41 of University Lecture Series.

[Hag11] J. Haglund. A polynomial expression for the Hilbert series of the quotient ring of diagonal coinvariants. Adv. in Math., 227:2092-2106, 2011.

[Hag14] J. Haglund, March 25, 2014. Talk at Bijective and Algebraic Combinatorics Conference In Honor of Bruce Sagan's 60th Birthday.

[Hai01] M. Haiman. Hilbert schemes, polygraphs, and the Macdonald positivity conjecture. J. Amer. Math. Soc., 14:941-1006, 2001.

[Hai02] M. Haiman. Vanishing theorems and character formulas for the Hilbert scheme of points in the plane. Invent. Math., 149:371-407, 2002.

[HHL05a] J. Haglund, M. Haiman, and N. Loehr. A combinatorial formula for Macdonald polynomials. J. Amer. Math. Soc., 18:735-761, 2005.

$\left[\mathrm{HHL}^{+}\right.$05b] J. Haglund, M. Haiman, N. Loehr, J. B. Remmel, and A. Ulyanov. A combinatorial formula for the character of the diagonal coinvariants. Duke Math. J., 126:195-232, 2005.

[Lev12] P. Levande. Combinatorial Structures and Generating Functions of Fishburn Numbers, Parking Functions, and Tesler Matrices. PhD thesis, U. of Penn., 2012.

[Mac95] I. Macdonald. Symmetric Functions and Hall Polynomials. Oxford University Press, second edition, 1995.

[MMR14] K. Mészáros, A. H. Morales, and B. Rhoades. The polytope of Tesler matrices. arXiv:1409.8566, September 2014.

[RW13] J. B. Remmel and A. T. Wilson. An extension of MacMahon's equidistribution theorem to ordered set partitions. Preprint available at sites.google.com/site/ andywilsonmath/research, 2013.

[Sta99] R. P. Stanley. Enumerative Combinatorics, volume 2. Cambridge University Press, 1999. 